\title{
Mental health at the age of coronavirus: time for change
}

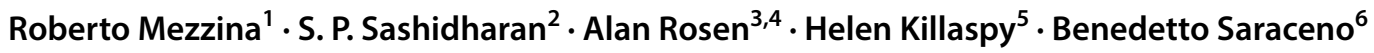

Received: 7 April 2020 / Accepted: 15 May 2020 / Published online: 29 May 2020

(c) Springer-Verlag GmbH Germany, part of Springer Nature 2020

\section{Introduction}

We are living in the age of the coronavirus (Covid-19). This emergency has put everything else in the background, conditioning and changing most aspects of our lives. As widely reported, health services in many countries are under enormous pressure and face unprecedented challenges. They are engaged in a heroic struggle but still suffer high death rates. With the spotlight on general health, mental health care suffers in silence. The need for mental health services has never been greater and, sooner or later, most of the population will experience increased discomfort and stress, particularly those with existing mental health problems. We must rethink mental health and mental health care in the era of coronavirus.

\section{Impact on mental health}

Franco Basaglia defined the 'social body' as the sum of subjects who participate in their own social organisation and responses to their needs and those of the group. Faced with an unprecedented crisis in health care generated by

Roberto Mezzina

romezzin@gmail.com

1 International School Franca and Franco Basaglia "The Practice of Freedom" and Former Director of the WHO Collaborating Centre, DSM, Trieste, Italy

2 Institute of Health \& Wellbeing, University of Glasgow, Glasgow, UK

3 Institute of Mental Health, University of Wollongong, Wollongong, Australia

4 Brain and Mind Centre, University of Sydney, Sydney, Australia

5 Division of Psychiatry, University College and Camden and Islington Mental Health NHS Foundation Trust, London, UK

6 School of Medical Sciences, University NOVA of Lisbon, Lisbon, Portugal
Covid-19, this 'social body' appears to be withdrawing in the face of emergency policies while the 'individual physical body' is positioned in the foreground [1].

Covid-19 is invading not only the 'individual physical body' but the 'social body'. Basaglia envisaged the 'restitution of the social body' of people who are excluded and marginalized as the utopia for modern mental health reform. [2] This ideal is now under threat as social networks shrink to the essential. We do not know to what extent social support is (or can be?) provided within these constraints. People are exhorted to distance themselves from the social body and social contacts are reduced to connections on the internet, telephone or TV. This, in turn, generates increasing exposure to unreliable sources, fear of exploitation and perceived loss of security, amplifying the experience of stress and suspicion.

The current crisis is predicted to last for some time, transforming individual and collective behaviours and habits. While the mental health impact of this is largely unknown, we do know from other epidemics like SARS that individual behaviours are shaped by extreme circumstances such as the psychological impact of quarantine [3]. With Covid-19, people who are segregated and quarantined are not just individuals who test positive for infection, but also those who are exposed to contagion and likely to develop serious health consequences as a result. The lockdown, whereby most of the population are confined to their homes, amounts to a huge collective experiment. It is a new 'norm', a generalised condition affecting everyone, at home, in health care facilities and all residential settings. The imperatives for social distancing and quarantine amount to societally sanctioned forms of involuntary seclusion and restraint, more usually seen in psychiatric hospitals.

In such circumstances, we must consider what can be done to improve people's mental health, from the most vulnerable to those at lowest risk. It is recognised that situations similar to lockdown can increase boredom, frustration, anxiety and even panic as we try to equip ourselves in the face of a danger which is strong and present, although invisible [4]. Some may resort to denial and escape from reality, 
obstinately maintaining their habitual lifestyle 'despite everything'. But, above all, long-term isolation can foster a condition of generalised trauma, with potential short-term as well as long-term consequences. Faced with an invisible contagion that you acquire and pass on as a consequence of your own behaviour, the social perception of disease becomes distorted through blame and guilt, both individual and collective. This can trigger unjustified forms of prejudice and stigma against individuals (who have been sick) or entire populations or particular groups [4].

\section{Impact on mental health services}

Services, such as outpatient clinics with often crowded waiting rooms and busy inpatient settings, are currently under review. For example, in Lombardy, Italy, during the sudden growth of the epidemic, acute hospital units were urgently converted to Covid-19 services with staff re-designated to work in this area. All specialist outpatient services are severely restricted and by appointment only - the antithesis of accessible, flexible services for a distressed population. Key principles of effective mental health care, such as close engagement and involvement with clients, are at risk because of the axiomatic rule of 'keep a distance'. The individual relationship, the main instrument of therapy and assistance in mental health, can no longer be used freely and directly.

The new priority for many workers in the mental health system is to identify and support people with this infection by providing relevant help, including psychological and psychiatric support. Yet the risk of contagion does not distinguish between who cares and who is cared for. Paradoxically, the health worker may threaten a patient's health as adequate precautions, such as elementary medical-surgical devices, masks, protective clothing and disinfectants are not always available in healthcare settings, whether in hospital, health centre or home.

Close therapeutic relationships are likely to be increasingly constrained and, finally, eliminated. Within a therapeutic relationship, the body is the medium of establishing contact and providing comfort. It is both individual and social at the same time. However, in the age of contagion, touching the body, the site where anguish is expressed and released, is inhibited as the 'organic body' becomes a source of danger [2].

As a result of the Covid-19 crisis, there is a risk that services providing personal, home-based support and vocational assistance will slow down or simply stop. The social, therapeutic and rehabilitative values of community mental health services are under threat because of the new emphasis on restrained, guarded social spaces. This reveals the fragility of a mental health system built on clinical 'places'. Hospital wards, emergency rooms and casualty departments have become sources of infection and places of contagion. Similarly, extra-hospital environments, the normal social spaces of the community and common life, are also sites of possible infection due to their normative functions, such as social meeting and exchange. This includes mental health facilities and communities where people live together, such as mental health residential care and supported housing. Already, we are seeing outbreaks of infection involving health care workers and guests in some care homes. However, national guidance seems slow in catching onto the need to provide tailored consideration of how to respond to the crisis in these settings. The size of the facility and style of management appear to be crucial factors: small group homes run as families, sharing a common view and approach with their guests, are, in the main, safe spaces, while larger residential facilities - more institution-like-such as elderly nursing homes are suffering. This is a general lesson to be learnt here: moving from standardized, institutional structures to personalized, tailored approach and services.

\section{Impact on people with pre-existing mental health problems}

Social detachment hangs over those who cannot or choose not to defend themselves in the prescribed way. There is a risk of greater self-isolation for those who are unable to comply or cope with emergency conditions and their vulnerabilities may be exacerbated during prolonged social isolation that 'lockdown' entails. Ironically, the social isolation sometimes seen in people with psychotic problems is now being normalised as part of the social behaviour imposed on everyone. This is the 'new normal'. Some individuals with serious mental health problems have also disappeared from services, holed up in houses, hospitals, supported accommodation and prisons. However, both the alienation generated by the absence of social ties and the exacerbation of conflict experienced within families can sharpen and lead to moments of crisis.

The rights and protection afforded to the most vulnerable in society are threatened as attempts to contain the spread of Covid-19 gather speed [5]. While this is understandable in the context of containing the pandemic, we cannot ignore the impact of such measures on basic human rights for all citizens. Well-intentioned prohibitions in extreme circumstances can easily become normative-resulting in a paranoid form of asocial regression and a form of 'mass hibernation'. At the same time, how can social distancing and self-isolation be enforced in a humane manner for those who cannot fully understand its importance to the survival of the population as well as the individual? Maintaining proximity and familiarity in everyday life, whether through intimacy or enforced closeness, may still be required. In the 
era of coronavirus, individual choices around how to mediate personal and familial boundaries may become severely circumscribed.

While most countries have acknowledged that the Covid19 outbreak and current strategies to combat the infection may have an adverse impact, they do not explicitly include the potentially harmful consequences for those with preexisting mental health conditions. Those who have always been marginalised in society due to their mental health problems continue to be ignored and forgotten. There is no doubt that basic human rights and the day-to-day environment of people with mental disorders are at risk. It is worth remembering that during the war in Iraq, patients of Baghdad psychiatric hospital were not only exposed to aerial bombing but were completely abandoned [6].

\section{Moving forward}

We need to prioritise services and identify those who need them most. This can be understood as selective prevention. High-risk groups include patients with delusions, obsessive-compulsive thoughts and behaviours, somatic symptoms, or those previously exposed to severe trauma [7]. Diagnosis by itself is a poor predictor of vulnerability. In identifying those at high risk, we need to consider a range of social factors and determinants of mental health and illmental health as well as subjective reactions based on individual life stories. For example, in countries like the US, a large number of people with serious mental illness are in jails, nursing and group homes or are homeless with limited access to medical and psychiatric care. They may be exposed to high risks. More frequent contact may be necessary for these groups to help them deal with emerging concerns and avoid serious exacerbation of existing problems or hospitalisation. People who are most vulnerable-not only in the medical or psychiatric sense, but socially—should be reached by telephone. Having a landline or a mobile phone is now recognized as a necessity for health so that electronic stores are allowed to stay open. Such contact may be as important as medication and help to contain the risks associated with quarantine and lockdown. The key to providing support is more extensive teleworking, with an emphasis on fostering relationships and a proxy sense of family and home, a sort of remote 'tele-heart' and 'tele-hearth'. In building and maintaining contact with these vulnerable groups, it is not just mental health but individual narratives and social situations that should be kept in mind.

The social gradient of mental health care is reinforced in the current crisis. The discomfort of the poorest, of those who are alone or confined in restricted spaces or in unsafe and inhumane settings, as the migrant population, may be exacerbated. Many will not have food or meals provided and may be unable to access social canteens. We need to ensure that basic needs are met. Homeless people living with mental illnesses are among the most vulnerable, lost in a social nothingness, without direction, and with even less support than normal since there are fewer people on the streets. Despite the efforts of the volunteer sector, they may be without shelter, basic medication, sanitation and hot food. Here, services must increase outreach care for those who cannot access them and provide appropriate support to ensure their survival. This will involve mobilising the available resources of communities, neighbourhoods, associations and churches.

It is important to find and share meaning in quarantine, beyond the rules of hygiene. The available guidance is largely limited to simple advice for those enduring various forms of quarantine; maintaining restful sleep, eating regular meals, exercise, limiting the use of alcohol, tobacco and other drugs, talking to loved ones about worries and fears, practicing relaxation strategies, engaging in hobbies and pleasant activities. There are also tips targeted at families, those with dependants or caring for older people [7]. At best, social and familial ties may be strengthened in times of danger, with families keeping and helping the sick to recover. People may also be looking outside their closed social networks and groups for comfort and support. For example, in many countries, local communities are coming together in support and solidarity, with an increase in charitable acts and a greater willingness to help others, albeit under highly constrained circumstances.

While more information, practical and emotional support and reassurance should be provided, it is also necessary to give meaning to isolation. This applies not merely to the vulnerable, but all of us. The collective search for meaning is a fundamental element of universal prevention. It concerns the whole population. This element will be decisive in the collective recovery phase that is coming.

\section{Learning from Covid 19}

If we look at what is happening, it is not sufficient to focus on physical health, for example fitness and healthy lifestyle. We also need to pay attention to a form of Foucauldian 'care of the self' [8]. What do we really need? What is essential? As we are thrown back on ourselves, something quite new to many of us, we must seize the opportunity to listen to ourselves and, at the same time, reinvigorate a sense of community and common struggle. This may foster a sense of collective heroism, where the fragmented and mediated 'social body' reconnects with multiple forms of support and survival. This is about the mental health of an entire society and a society is judged on how it treats its most vulnerable. 
We must defend mental health services, which interpret and mediate this 'social body', while defending ourselves.

The most insidious effects of the outbreak on mental health will not be apparent immediately. These are more likely to manifest after the loosening of the current lockdown and the resumption of normal life. It is then that the consequences of human and economic losses will emerge. To address this, we will need to engage communities, integrate professional interventions with community involvement, strengthen community-based interventions with a multidisciplinary and multisectoral approach and pool resources available for mental health support. In time, it will be important to identify and understand the factors associated with individual and collective resilience and the coping strategies that promote a collective, mass recovery. The "whole society approach' [9] suggests that new forms of social connection should be developed and enhanced as part of a collective effort to tackle the social problems thrown up by Covid-19.

We need to avoid fragmentation of our efforts by building alliances between public mental healthcare, social services and the third sector. This will ensure an effective response to whole life needs, protecting not only health but also human rights of people living in institutions, hospitals, prisons, shelters, nursing homes, group homes and other special facilities, which means acting for their deinstitutionalization. For those experiencing social deprivation, this will mean increasing access to care. It is important to implement the emergency national plans dedicated to mental health in the affected countries, called for the World Health Organization and reiterated by the World Federation for Mental Health [10], and most recently prompted by the United Nations [11]. It has never been more urgent to step aside from individualistic notions and embrace the values and practices of sharing and solidarity, both civil and social. This will enhance our sense of being part of a community. Mental health services, which have a long history of community networking and engagement, can act as exemplars and provide essential bridges to a 'new normal', with collaboration and shared responsibilities for each other at its heart.

\section{References}

1. Basaglia F (1987) Madness/Delirium. In: Hughes NS, Lowell A (eds) Psychiatry inside out. Selected writings of Franco Basaglia. Columbia University Press, New York, pp 231-263

2. Mezzina R (2020) Mental health services, individuals and the 'social body' at the time of the coronavirus. https://imhen.org/

3. Manderscheid RW (2007) Preparing for pandemic Avian Influenza: ensuring mental health services and mitigating panic. Arch Psychiatr Nurs 21(1):64-67

4. Brooks SK, Webster RK, Smith LE, Woodland L, Wessely S, Greenberg N, Rubin GJ (2020) The psychological impact of quarantine and how to reduce it: rapid review of the evidence. Lancet 395:912-920. https://doi.org/10.1016/S0140-6736(20)30460-8

5. https://www.hsj.co.uk/coronavirus/government-to-make-emerg ency-changes-to-mental-health-act/7027149.article

6. Van Ommeren M, Saxena S, Loretti A, Saraceno B (2003) Ensuring care for patients in custodial psychiatric hospitals in emergencies. The Lancet 362:574

7. American Psychiatric Association (APA). COVID-19 mental health impacts: resources for psychiatrists. https://www.psych iatry.org/news-room/apa-blogs/apa-blog/2020/03/covid-19-menta 1-health-impacts-resources-for-psychiatrists

8. McGushin E (2011) Foucault's theory and practice of subjectivity. In: Taylor D (ed) Michel Foucault: key concepts. Acumen Publishing Ltd., Durham, pp 127-142

9. United Nations Inter Agency Standing Committee (IASC) (2020) Psychosocial support in emergency settings. Briefing note on addressing mental health and psychosocial aspects of COVID-19 outbreak-Version 1.0

10. The World Federation for Mental Health (2020) Appeal for National Plans for Mental Health during the Coronavirus Global Emergency. https://wfmh.global/mental-health-and-covid-19appeal/

11. UN Secretary-General Policy Brief: COVID-19 and the need for action on mental health. https://www.un.org/sites/un2.un.org/files /un_policy_brief-covid_and_mental_health_final.pdf 